\title{
Management of Pregnancy-Associated Thrombotic Thrombocytopenia Purpura
}

\author{
Ashley Fyfe-Brown, MD ${ }^{1}$ Gwen Clarke, MD $\quad$ Kara Nerenberg, MD, MSc ${ }^{3} \quad$ Sujata Chandra, MD, MSc ${ }^{1}$ \\ Venu Jain, $\mathrm{MD}, \mathrm{PhD}^{1}$
}

${ }^{1}$ Division of Maternal-Fetal Medicine, Royal Alexandra Hospital, University of Alberta, Edmonton, Alberta, Canada

2 Division of Hematology, Royal Alexandra Hospital, University of Alberta, Edmonton, Alberta, Canada

3 Division of General Internal Medicine, Royal Alexandra Hospital, University of Alberta, Edmonton, Alberta, Canada

\begin{abstract}
Address for correspondence Venu Jain, Assistant Professor, MD, PhD, 5S131 Lois Hole Hospital, Royal Alexandra Hospital, 10240 Kingsway Avenue, Edmonton, Alberta T5H 3V9, Canada (e-mail: venu.jain@albertahealthservices.ca).
\end{abstract}

Am J Perinatol Rep 2013;3:45-50.

\author{
Abstract \\ Keywords \\ - fresh frozen plasma \\ - cryosupernatant \\ plasma \\ - platelet transfusion \\ - preeclampsia \\ - HELLP syndrome \\ - TTP
}

Thrombotic thrombocytopenia purpura (TTP) is an infrequent but serious disease. Pregnancy is a known risk factor for presentation or relapse of TTP. Difficulties in differentiating TTP from preeclampsia/HELLP (hemolysis, elevated liver enzymes and low platelets) syndrome, and current treatment recommendations are discussed in this case report. A woman with previously treated and stable TTP had a relapse at 36 weeks' gestation. Careful surveillance led to an early diagnosis. Severe disease in the peripartum period was treated successfully with cryosupernatant plasma-based plasmapheresis and platelet transfusion, with good maternal and neonatal outcomes. Cryosupernatant plasma is a viable alternative to fresh frozen plasma for plasmapheresis for TTP and may offer some therapeutic and logistical advantages. Platelet transfusion can be undertaken safely if needed to prevent or treat significant hemorrhage.
Thrombotic thrombocytopenic purpura (TTP) is an uncommon and potentially fatal disorder that may affect women of childbearing age. ${ }^{1-3}$ The pentad of clinical features associated with TTP includes microangiopathic hemolytic anemia, thrombocytopenia, neurological and renal abnormalities, and fever. ${ }^{1,2}$ It is caused by absent or severely depleted "von Willebrand factor (vWF) cleaving protease" (a disintegrin and metalloprotease with thrombospondin1-like domains, or ADAMTS-13). ${ }^{4,5}$ This enzyme is needed for cleavage of the ultralarge vWF multimers produced by the vascular endothelium to a smaller multimer normally present in the circulation. A congenital or acquired deficiency of ADAMTS13 can result in accumulation of the ultralarge vWF multimers leading to microangiopathic thromboses and severe thrombocytopenia with resultant hemolytic anemia and thrombotic sequelae. ${ }^{6}$ In the rare familial forms of TTP, mutations of the ADAMTS-13 gene can be found resulting in significant risk of TTP in pregnancy in affected women. ${ }^{6}$

received

August 12, 2012

accepted after revision

August 14, 2012

published online

December 19, 2012
Acquired TTP is postulated to be secondary to viral infections that trigger production of antibodies targeted against ADAMTS-13. ${ }^{7}$ Women with acquired TTP also have a high risk of recurrence in a subsequent pregnancy. ${ }^{6}$ It has been proposed that specific proteins found in the placental circulation serve as antigens that trigger maternal antibody production against ADAMTS-13. As such, TTP associated with pregnancy accounts for 12 to $31 \%$ of all cases of TTP and is associated with high rates of obstetric complications. $^{8-10}$ Untreated, TTP is associated with a $>90 \%$ mortality. ${ }^{2,11}$

This case serves to describe the clinical course and management of TTP in a primigravid patient with a known prior episode of thrombocytopenic purpura. Specific objectives for this case report include: first, the differentiation of TTP from preeclampsia/HELLP (hemolysis, elevated liver enzymes and low platelets) syndrome in pregnancy; and second, a review of the literature on the current recommendations for
Copyright $\odot 2013$ by Thieme Medical Publishers, Inc., 333 Seventh Avenue, New York, NY 10001, USA. Tel: +1(212) 584-4662.
DOI http://dx.doi.org/ $10.1055 / \mathrm{s}-0032-1331380$. ISSN 2157-6998. 
management of TTP in pregnancy, in particular the use of cryosupernatant plasma (CSP) and platelets.

\section{Case Report}

Our multidisciplinary team (obstetrics, maternal-fetal medicine, obstetric medicine, hematology, and endocrinology) followed a 32-year-old primigravida throughout her pregnancy for several medical issues including a previous history of TTP, chronic hypertension, hypothyroidism, obesity, and gestational diabetes.

The patient had been diagnosed with TTP approximately 8 months prior to conception. At that time, her presenting symptoms were abdominal pain with bloody diarrhea, fevers, and malaise. On exam, she was febrile and had numerous petechiae. Her platelet count was $5 \times 10^{9} / \mathrm{L}$, and a microangiopathic hemolytic anemia was confirmed. The TTP was thought to be acquired though no clear etiology was found despite extensive testing. She was treated with 14 courses of plasmapheresis and prednisone. Low-dose aspirin was also initiated. Following treatment, her laboratory values stabilized (platelet count $>200 \times 10^{9} / \mathrm{L}$ ) and her ADAMTS-13 activity was $40 \%$. Despite a relatively elevated level of ADAMTS-13, she was advised by her hematologist to postpone a pregnancy for approximately 2 years given the high risk of recurrence of TTP during pregnancy.

The patient subsequently conceived and her medical issues were stable during the pregnancy. She remained clinically and biochemically euthyroid on $0.05 \mathrm{mg} / \mathrm{d}$ of thyroxine replacement. Her blood pressure was well controlled on labetalol $200 \mathrm{mg}$ twice a day with no features of preeclampsia. She developed gestational diabetes, which was adequately controlled with insulin. She was monitored with biweekly investigations including complete blood counts, lactate dehydrogenase (LDH) and bilirubin levels. Her ADAMTS-13 activity at approximately 20 weeks was $45 \%$, which was thought not to be predictive of relapse. At a routine obstetric medicine visit at $36^{+5}$ weeks' gestation, the patient appeared unwell. She reported epistaxis with sneezing, one bloody bowel movement, and "tea-colored" urine. Her blood pressure was elevated $(150 / 100 \mathrm{~mm} \mathrm{Hg})$ with mild peripheral edema. Her abdomen and extremities were covered in petechiae (-Fig. 1). Her neurological examination was normal. The patient was immediately admitted to the obstetric service for investigations for HELLP syndrome versus TTP (day 0, - Fig. 2). Initial investigations were remarkable for platelets critically low at $6 \times 10^{9} / \mathrm{L}$, hemoglobin $117 \mathrm{~g} / \mathrm{L}, \mathrm{LDH} 1182 \mathrm{U} / \mathrm{L}$, bilirubin $32 \mu \mathrm{mol} / \mathrm{L}$, and aspartate aminotransferase (AST) 50 U/L (-Fig. 2). Peripheral smear showed severe hemolysis with schistocytes. There was $3+$ proteinuria on urine dipstick. Fetal ultrasound revealed a singleton fetus with normal growth, no structural anomalies, and oligohydramnios (amniotic fluid index of $3.8 \mathrm{~cm}$ ). After multidisciplinary consultation (hematology, hematopathology, obstetric medicine, maternal-fetal medicine, and intensive care), the working diagnosis was TTP and plasmapheresis was urgently initiated in the intensive care setting. Plasmapheresis consisted of $1.5 \times$ plasma volume exchange per day using the patient's

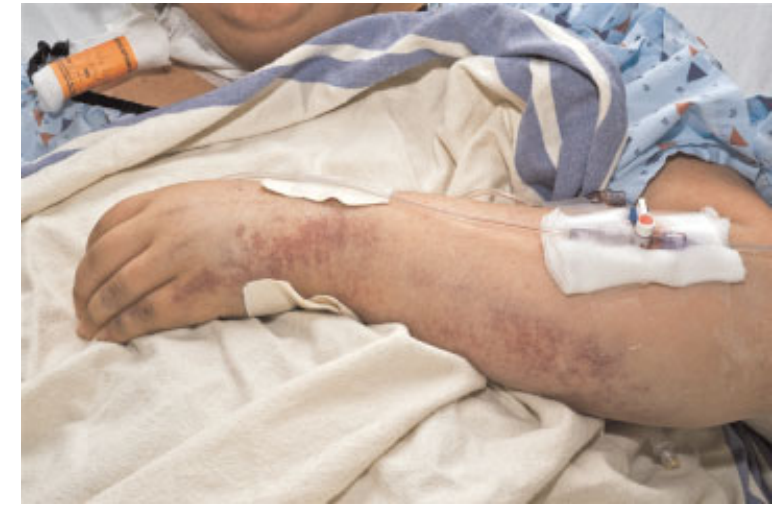

Fig. 1 Purpuric rash on forearm and multiple vascular access lines in a patient with thrombotic thrombocytopenia purpura during pregnancy.

blood pressure, urine output, and fetal heart rate monitoring to guide the rate of plasmapheresis and ensure an optimal rate of exchange (-Fig. 2). CSP was used for all treatments. Other TTP treatments included prednisone $125 \mathrm{mg} / \mathrm{d}$ ( $1 \mathrm{mg} / \mathrm{kg}$ ) and aspirin $81 \mathrm{mg} / \mathrm{d}$. Blood pressure was controlled with increasing doses of labetalol.

The third day following admission (day 2, - Fig. 2), at 37 weeks, the fetal heart rate monitoring was nonreassuring with reduced variability and variable decelerations. Given the concern regarding fetal heart rate pattern with known oligohydramnios, after a multidisciplinary team discussion, the baby was delivered by cesarean section. By this time, the patient's platelet level had increased to only $40 \times 10^{9} / \mathrm{L}$, therefore the anesthesia team managed the patient with an awake intubation followed by a general anesthetic. A female infant weighing $2818 \mathrm{~g}$ was delivered, with an Apgar score of 2,5 , and 8 at 1,5 , and 10 minutes, respectively. Due to excessive intraoperative bleeding, two doses of platelets (one of apheresis platelets and one of pooled platelets) and $3 \mathrm{U}$ of packed red blood cells were infused. Her postoperative

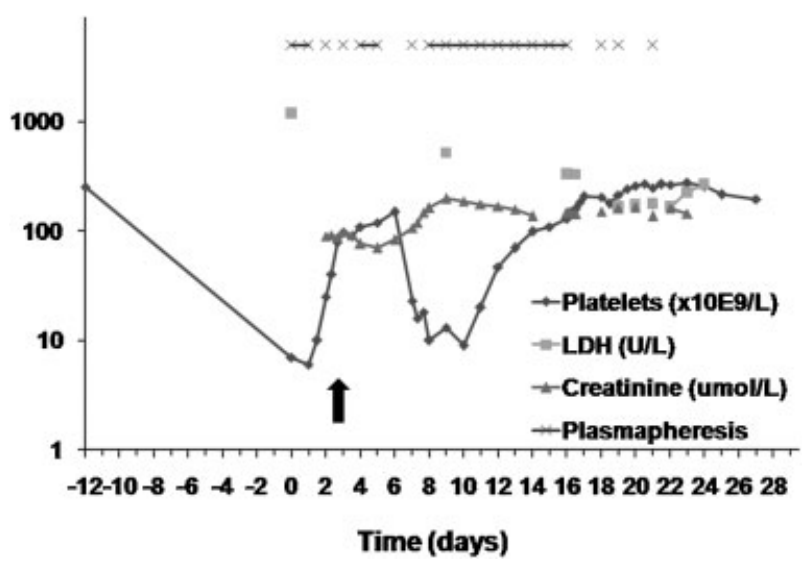

Fig. 2 Hematologic and biochemical changes during the course of thrombotic thrombocytopenia purpura and timing of plasmapheresis. Day 0 is the day of hospital admission. The arrow indicates the timing of intraoperative platelet transfusion on day 2 . The y-axis is in log scale. $\mathrm{LDH}$, lactate dehydrogenase. 
hemoglobin was $95 \mathrm{~g} / \mathrm{L}$. Plasmapheresis was continued after the cesarean section.

The postoperative course was complicated by significant hypertension and nephrotic-range proteinuria (peak $13 \mathrm{~g}$ in 24 hours), likely secondary to concurrent preeclampsia, requiring magnesium sulfate and progressive increases in four antihypertensive agents (labetalol, nifedipine, hydralazine, and clonidine). She developed acute renal injury with a peak creatinine of $220 \mu \mathrm{mol} / \mathrm{L}$. Nephrology was consulted and after extensive investigations, the cause was attributed to TTP with microvascular infarctions. Additional postoperative complications included blurred vision secondary to bilateral choroidal infarcts from TTP and severe hypertension, and also a cesarean section wound infection requiring intravenous antibiotics. Wound culture identified Escherichia coli as the causal organism.

This patient required a total of 24 cycles of plasmapheresis and a prolonged course of prednisone. Four months postpartum, her platelets remained stable at $250 \times 10^{9} / \mathrm{L}$ and hemoglobin was $118 \mathrm{~g} / \mathrm{L}$. Her renal dysfunction, proteinuria, and dysglycemia had resolved. Her blood pressure remained stable on high doses of four antihypertensive medications. She remains on aspirin $81 \mathrm{mg}$ daily for prevention of TTP. She agreed for the placement of an intrauterine contraceptive device for contraception.

\section{Discussion}

This case illustrates the complex clinical features that may accompany a diagnosis of microangiopathic hemolysis and the difficulty in distinguishing TTP from HELLP syndrome, even in a patient with a known prior history of TTP. Differentiation of these two conditions is critical as the treatment of HELLP syndrome differs substantially from that of TTP. Although plasmapheresis is the mainstay of therapy in TTP, delivery of the infant is usually required in HELLP syndrome to reverse the course of the disease. The distinction between TTP and HELLP syndrome can be challenging given the overlap of several features (microangiopathic hemolytic anemia and thrombocytopenia). Easy bruising is often the first reliable indication of the development of TTP, with the majority of cases presenting in the second trimester and $33 \%$ presenting after 28 weeks. ${ }^{3}$ Features that may help to distinguish TTP include renal dysfunction, significantly reduced platelet counts (less the 20,000), fever, and fluctuating neurological symptoms. ${ }^{1-3}$ In addition, very high elevations of LDH with only moderate elevations of AST resulting in an elevated LDH-to-AST ratio suggest TTP. ${ }^{3}$ Although reduced ADAMTS-13 activity and elevated vWF levels can be seen in HELLP syndrome, a severe reduction in ADAMTS-13 activity along with an increase in ultralarge vWF multimers is usually suggestive of TTP. ${ }^{12,13}$ Severe thrombocytopenia in HELLP syndrome usually responds to steroids within 8 to 12 hours; therefore, lack of this response would also favor a diagnosis of TTP. $^{3}$ Given that HELLP syndrome is often associated with preeclampsia, maternal hypertension and proteinuria would favor a diagnosis of HELLP syndrome. However, preeclampsia/ HELLP syndrome occurs concurrently in $17 \%$ cases of TTP during pregnancy. This results in a significantly increased maternal mortality of $44 \%$ as opposed to a $25 \%$ overall mortality with pregnancy-associated TTP.

TTP is associated with an increased risk of stillbirth. With availability of therapy, the stillbirth rate had decreased from 69 to $45 \%{ }^{3}$ More losses occur in the second trimester, and the third-trimester loss rate has declined from 50 to $17 \%$. Of note, TTP does not increase the risk of thrombocytopenia in the fetus/newborn. ${ }^{14}$

Patients with a history of TTP who subsequently become pregnant represent a group at high risk of TTP recurrence. Maternal mortality is higher (26\%) in women who present with their initial episode in pregnancy versus those with recurrent disease (11\%). ${ }^{3}$ It is unclear whether this higher mortality is a result of a delay in diagnosis. Risk of recurrence of TTP in a future pregnancy is $92 \%$ in women with congenital TTP, $47 \%$ in acquired idiopathic TTP, and 45\% in acquired pregnancy-associated TTP. ${ }^{6}$ Given the increased risk of recurrence in pregnancy, women with a previous history of TTP should be monitored closely for the development of TTP. In addition to regular investigations for evidence of thrombocytopenia and microangiopathic hemolysis, serial measurement of ADAMTS-13 activity can be used to assist in the early diagnosis of a recurrence. ${ }^{9,10,15}$ Although severe ADAMTS-13 deficiency ( $<2.5 \%$ of normal activity) may be helpful in classifying low versus high risk of TTP recurrence, as evidenced by the current case, this level may not be sensitive enough to identify patients at risk of relapse of TTP. We suggest clinical monitoring along with serial measurements of ADAMTS-13 activity, complete blood count, and LDH levels and very close clinical follow-up of patients with ADAMTS-13 activity $<10 \%$. There is uncertainty as to the cutoff of ADAMTS-13 level to initiate therapy. ADAMTS-13 activity decreases during pregnancy in normal women., 9,15 The levels are normal in the first trimester, but may drop as low as 25 to $30 \%$ of the normal range in the second and third trimesters. Scully et al proposed a $5 \%$ cutoff for ADAMTS- 13 activity for the decision to institute plasma-based therapy. ${ }^{9}$ Raman et al recommend that ADAMTS-13 activity $<2.5 \%$ during pregnancy is predictive of a high risk of recurrence and would justify prophylactic plasma-based therapy. ${ }^{10}$ Their recommendation applies whether the initial episode of TTP was idiopathic or pregnancy-related. Given the limited number of women with TTP, the optimal level of ADAMTS-13 activity to initiate plasmapheresis is difficult to study in a clinical trial setting.

Plasma-based therapy is the mainstay of treatment for TTP. ${ }^{11,16}$ Plasmapheresis was developed in the 1970 s and represented a major advance in the treatment of TTP. ${ }^{15,17}$ This therapy is thought to replenish the depleted levels of ADAMTS-13, thus reducing the thrombotic microangiopathy. Plasma exchange has been shown to be more effective than plasma infusion when overall survival rates were compared between the two groups. ${ }^{11}$ It also allows delivery of three times the plasma compared with infusion alone, with obvious benefits to the patient. Plasmapheresis should be continued for at least 2 days after the achievement of remission as defined by normal platelet count and LDH levels and rising 
hemoglobin. At this time, fresh frozen plasma (FFP) is considered to be the optimal replacement fluid. ${ }^{11,15} \mathrm{CSP}$, the supernatant resulting from production of cryoprecipitate, is used in some centers for treatment of TTP. ${ }^{11,18,19}$ CSP has been shown to have similar levels of ADAMTS-13 activity when compared with FFP. However, most of vWF, especially the ultralarge multimers, distributes into the cryoprecipitate, with little remaining in CSP. Theoretically, CSP is ideal for therapy because of adequacy of the therapeutic ADAMT-13 levels without significant amounts of its pathogenic substrate (i.e., vWF). CSP has been found to be as effective as FFP for treatment of TTP, ${ }^{11,18,19}$ with some studies reporting higher efficacy of CSP. ${ }^{20,21}$ Patients with TTP often need infusion of large volumes of plasma. Therefore, from a practical standpoint, use of CSP in this population allows FFP and cryoprecipitate to remain available for other patients needing replacement of multiple coagulation factors.

The use of platelet transfusions in patients with TTP is controversial. There have been reports of sudden deterioration associated with their use, thought to be a result of increased formation of microthrombi. ${ }^{22}$ However, a more recent review by Swisher et al could not demonstrate evidence of harm from platelet transfusion in patients with TTP. ${ }^{23}$ Current recommendations are that platelet transfusion should only be used to prevent or manage life-threatening hemorrhage. These principles are illustrated in this case, with platelets used only for prevention of hemorrhage at the time of cesarean section.

Low-dose aspirin therapy is used in patients with prior TTP to prevent recurrences. ${ }^{3,24}$ However, there is a paucity of data from prospective, randomized controlled trials on the effectiveness of this approach. ${ }^{25}$ Low-molecular-weight heparin also has been suggested as an effective preventative approach $^{2,8,9}$; however there is little supporting clinical evidence available at this time. High-dose corticosteroid therapy and other immunosuppressive treatments have been reserved for patients with severe TTP on clinical presentation or an ADAMTS- 13 level $<10 \%$ of normal. ${ }^{2}$

In summary, TTP in pregnancy is a rare but serious disease with high rates of maternal and perinatal morbidity and mortality. Clinical signs and symptoms of TTP with corroborating laboratory evidence of microangiopathic hemolytic anemia and thrombocytopenia remain the mainstay of the clinical diagnosis of TTP in pregnancy and help to distinguish TTP from preeclampsia/HELLP syndrome. ADAMTS-13 activity can also be useful in early identification of patients with recurrent TTP. A diagnosis of TTP necessitates urgent and aggressive plasma-based treatment. The decision regarding when to deliver should be made on clinical grounds that include gestational age, response to therapy, and fetal condition. ${ }^{9,26}$

\section{References}

1 George JN. Clinical practice. Thrombotic thrombocytopenic purpura. N Engl J Med 2006;354:1927-1935

2 George JN. How I treat patients with thrombotic thrombocytopenic purpura: 2010. Blood 2010;116:4060-4069
3 Martin JN Jr, Bailey AP, Rehberg JF, Owens MT, Keiser SD, May WL. Thrombotic thrombocytopenic purpura in 166 pregnancies: 1955-2006. Am J Obstet Gynecol 2008;199:98-104

4 Plaimauer B, Zimmermann K, Völkel D, et al. Cloning, expression, and functional characterization of the von Willebrand factorcleaving protease (ADAMTS13). Blood 2002;100:3626-3632

5 Remuzzi G, Galbusera M, Noris M, et al; Italian Registry of Recurrent and Familial HUS/TTP. Thrombotic thrombocytopenic purpura/hemolytic uremic syndrome. von Willebrand factor cleaving protease (ADAMTS13) is deficient in recurrent and familial thrombotic thrombocytopenic purpura and hemolytic uremic syndrome. Blood 2002;100:778-785

6 Vesely SK, Li X, McMinn JR, Terrell DR, George JN. Pregnancy outcomes after recovery from thrombotic thrombocytopenic purpura-hemolytic uremic syndrome. Transfusion 2004;44: 1149-1158

7 Rieger M, Mannucci PM, Kremer Hovinga JA, et al. ADAMTS13 autoantibodies in patients with thrombotic microangiopathies and other immunomediated diseases. Blood 2005;106:1262-1267

8 George JN. How I treat patients with thrombotic thrombocytopenic purpura-hemolytic uremic syndrome. Blood 2000;96: 1223-1229

9 Scully M, Starke R, Lee R, Mackie I, Machin S, Cohen H. Successful management of pregnancy in women with a history of thrombotic thrombocytopaenic purpura. Blood Coagul Fibrinolysis 2006;17: 459-463

10 Raman R, Yang S, Wu HM, Cataland SR. ADAMTS13 activity and the risk of thrombotic thrombocytopenic purpura relapse in pregnancy. Br J Haematol 2011;153:277-279

11 Michael M, Elliott EJ, Ridley GF, Hodson EM, Craig JC. Interventions for haemolytic uraemic syndrome and thrombotic thrombocytopenic purpura. Cochrane Database Syst Rev 2009;(1):CD003595

12 Lattuada A, Rossi E, Calzarossa C, Candolfi R, Mannucci PM. Mild to moderate reduction of a von Willebrand factor cleaving protease (ADAMTS-13) in pregnant women with HELLP microangiopathic syndrome. Haematologica 2003;88:1029-1034

13 Molvarec A, Rigó JJr, Bõze T, et al. Increased plasma von Willebrand factor antigen levels but normal von Willebrand factor cleaving protease (ADAMTS13) activity in preeclampsia. Thromb Haemost 2009;101:305-311

14 Burrows RF, Kelton JG. Fetal thrombocytopenia and its relation to maternal thrombocytopenia. N Engl J Med 1993;329: 1463-1466

15 Scully M, Longair I, Flynn M, Berryman J, Machin SJ. Cryosupernatant and solvent detergent fresh-frozen plasma (Octaplas) usage at a single centre in acute thrombotic thrombocytopenic purpura. Vox Sang 2007;93:154-158

16 Fontana S, Kremer Hovinga JA, Lämmle B, Mansouri Taleghani B. Treatment of thrombotic thrombocytopenic purpura. Vox Sang 2006;90:245-254

17 Nguyen TC, Stegmayr BG, Busund R, Bunchman TE, Carcillo JA. Plasma therapies in thrombotic syndromes. Int J Artif Organs 2005;28:459-465

18 Altuntas F, Aydogdu I, Kabukcu S, et al. Therapeutic plasma exchange for the treatment of thrombotic thrombocytopenic purpura: a retrospective multicenter study. Transfus Apheresis Sci 2007;36:57-67

19 Byrnes JJ, Moake JL, Klug P, Periman P. Effectiveness of the cryosupernatant fraction of plasma in the treatment of refractory thrombotic thrombocytopenic purpura. Am J Hematol 1990;34: 169-174

20 Owens MR, Sweeney JD, Tahhan RH, Fortkolt P. Influence of type of exchange fluid on survival in therapeutic apheresis for thrombotic thrombocytopenic purpura. J Clin Apher 1995;10:178-182

21 Rock G, Shumak KH, Sutton DM, Buskard NA, Nair RC; Members of the Canadian Apheresis Group. Cryosupernatant as replacement fluid for plasma exchange in thrombotic thrombocytopenic purpura. Br J Haematol 1996;94:383-386 
22 Harkness DR, Byrnes JJ, Lian EC, Williams WD, Hensley GT. Hazard of platelet transfusion in thrombotic thrombocytopenic purpura. JAMA 1981;246:1931-1933

23 Swisher KK, Terrell DR, Vesely SK, Kremer Hovinga JA, Lämmle B, George JN. Clinical outcomes after platelet transfusions in patients with thrombotic thrombocytopenic purpura. Transfusion 2009; 49:873-887

24 Myers TJ, Wakem CJ, Ball ED, Tremont SJ. Thrombotic thrombocytopenic purpura: combined treatment with plasmapheresis and antiplatelet agents. Ann Intern Med 1980;92(2 Pt 1):149-155
25 Bobbio-Pallavicini E, Gugliotta L, Centurioni R, et al. Antiplatelet agents in thrombotic thrombocytopenic purpura (TTP). Results of a randomized multicenter trial by the Italian Cooperative Group for TTP. Haematologica 1997;82:429-435

26 He Y, Chen Y, Zhao Y, Zhang Y, Yang W. Clinical study on five cases of thrombotic thrombocytopenic purpura complicating pregnancy. Aust N Z J Obstet Gynaecol 2010;50:519-522 